\title{
Nanotheranostics
}

2018; 2(1): 1-11. doi: 10.7150/ntno.22335

Research Paper

\section{Dual radiosensitization and anti-STAT3 anti-prolifera- tive strategy based on delivery of gold nanoparticle - oligonucleotide nanoconstructs to head and neck cancer cells}

\author{
Surong Zhang ${ }^{1 凶}$, Suresh Gupta1 ${ }^{1}$, Thomas J Fitzgerald², Alexei A Bogdanov, Jr. ${ }^{1 凶}$ \\ 1. Laboratory of Molecular Imaging Probes, Department of Radiology, University of Massachusetts Medical School, Worcester MA, USA; \\ 2. Department of Radiation Oncology, University of Massachusetts Medical School, Worcester, MA, USA. \\ $\square$ Corresponding authors: S6-326, Department of Radiology, University of Massachusetts Medical School, 55 Lake Ave North, Worcester MA 01655 Tel. \\ 508-856-5571 FAX 508-856-1860 Surong.Zhang@umassmed.edu; Alexei.Bogdanov@umassmed.edu \\ (C) Ivyspring International Publisher. This is an open access article distributed under the terms of the Creative Commons Attribution (CC BY-NC) license \\ (https://creativecommons.org/licenses/by-nc/4.0/). See http://ivyspring.com/terms for full terms and conditions.
}

Received: 2017.08.11; Accepted: 2017.10.15; Published: 2018.01.01

\begin{abstract}
Constitutively activated signal transducer and activator of transcription 3 (STAT3) factor is an important therapeutic target in head and neck cancer (HNC). Despite early promising results, a reliable systemic delivery system for STAT3- targeted oligonucleotide (ODN) drugs is still needed for future clinical translation of anti-STAT3 therapies. We engineered and tested a novel ODN duplex/gold nanoparticle (AuNP)-based system carrying a therapeutic STAT3 decoy (STAT3d) payload. This strategy is two-pronged because of the additive STAT3 antagonism and radiosensitizing properties of AuNP. The specificity to head and neck cancer cell surface was imparted by using a nucleolin aptamer (NUAP) that was linked to AuNP for taking the advantage of an aberrant presentation of a nuclear protein nucleolin on the cell surface. STAT3d and nucleolin aptamer constructs were independently linked to AuNPs via Au-S bonds. The synthesized AuNP constructs (AuNP-NUAP-STAT3d) exhibited internalization in cells that was quantified by using radiolabeled STAT3d. AuNP-NUAP-STAT3d showed radiosensitizing effect in human HNC FaDu cell culture experiments that resulted in an increase of cell DNA damage as determined by measuring $\mathrm{Y}-\mathrm{H} 2 \mathrm{AX}$ phosphorylation levels by flow cytometry. The radiosensitization study also demonstrated that AuNP-NUAP-STAT3d as well as STAT3d alone resulted in the efficient inhibition of A431 cell proliferation. While FaDu cells did not show instant proliferation inhibition after incubating with AuNP-NUAP-STAT3d, the cell DNA damage in these cells showed nearly a $50 \%$ increase in AuNP-NUAP-STAT3d group after treating with radiation. Compared with anti-EGFR humanized antibody (Cetuximab), AuNP-NUAP-STAT3d system had an overall stronger radiosensitization effect in both $\mathrm{A} 431$ and $\mathrm{FaDu}$ cells.
\end{abstract}

Key words: oligonucleotide, aptamer, gold nanoparticle, confocal microscopy, STAT3.

\section{Introduction}

Despite recent progress in decreasing mortality rates, head and neck cancers, which constitute $2 \%$ of all cancers in women and $4 \%$ in men, an estimated 49,670 new cases of cancer of the oral cavity and pharynx (throat) (oral, lip and oropharyngeal cancer accounts for up to $75 \%$ of all HNC) are expected in
2017 in the USA [1]. The progression of HNC had been linked to constitutive activation of JAK/STAT3 axis via IGF and EGFR signaling that has been implicated in promoting resistance to apoptosis and immune evasion/suppression [2]. Signal transducer and activator of transcription 3 (STAT3) is a 
transcription factor that is constitutively activated in a variety of human malignancies, including prostate, lung, brain, breast, and squamous cell carcinomas and skin cancer [3, 4]. Constitutively activated STAT3 is an early event in head and neck carcinogenesis that contributes to the loss of growth control by an anti-apoptotic mechanism [5].

STAT3 is a critical regulating element of EGFR-STAT3-BclXL signaling axis, aberrantly activated in majority of HNC [6]. In the past, blocking of STAT3 activation with STAT3 decoys (STAT3d) has been actively explored as a potential strategy for attenuating resistance to chemo-, radio- and other therapies of HNC [2]. STAT3 decoy (STAT3d) is a therapeutic oligonucleotide duplex that potentially could be used to decrease STAT3 activation. STAT3d has shown selective binding for STAT3 protein and has been shown to inhibit the proliferation and survival of HNC [7] and the growth of HNC xenograft tumors in vivo [8]. Current limitation in the translation of STAT3d into clinical use is insufficient stability and a lack of specificity to HNC surface resulting in a low efficacy of systemic delivery to head and neck tumors. Gold nanoparticles for years were considered attractive as delivery agents and radiosensitizers due to past clinical use, the ease of surface modification and conjugation, high $Z(Z=79)$ of gold with strong absorption of $X$-ray as well as the subsequent emission of secondary electrons (such as Auger electrons, photoelectrons and Compton electrons) and enhanced localized radiation dosage [9-16]. We hypothesized that AuNPs due to a very large cumulative surface area are capable of delivering high numbers of STAT3d to tissues and into cells, and that the accumulation of AuNPs in cancer cells or tumor can enhance radiation treatment resulting in additive therapeutic effect.

Cancer-targeted therapies in most cases rely on cell surface receptors to direct anticancer agents to tumors [17, 18]. Therefore, we decided to explore known aberrant expression of nucleolin for enhancing targeted therapy to head and neck cancer cells which frequently display this protein on the surface $[19,20]$. We linked nucleolin aptamer to AuNP-STAT3d delivery system. Nucleolin aptamer is known to have high affinity to nucleolin, a Bcl-2 mRNA binding protein involved in cell proliferation [21, 22]. The overexpression of nucleolin on the cell surface and in the cytoplasm of exponentially growing cells suggests that this protein not only can shuttle molecules between the nucleus and the cytoplasm, but also can function as a receptor for cell-type independent targeted cancer treatment.

In this study, we report on a novel dual theranostic strategy in which the potential of AuNP is explored for both as a vehicle for STAT3d delivery via cell-surface nucleolin and simultaneously as a radiosensitizer for combinational therapy of head-and-neck cancer.

\section{Materials and Methods}

\section{Cell culture}

Human cervical carcinoma HeLa cell line (American Type Culture Collection, ATCC, CCL-2) was maintained in DMEM (Gibco ${ }^{\mathrm{TM}}$ DMEM, Thermo Fisher Scientific) supplemented with $10 \%$ fetal bovine serum, FBS (Thermo Fisher Scientific). The human epithelial cell line MCF-10A (ATCC, CRL-10317) was maintained in DMEM/F12 medium supplemented with $10 \%$ horse serum (Thermo Fisher Scientific), 20 $\mathrm{ng} / \mathrm{mL}$ epidermal growth factor (Sigma-Aldrich), 0.5 $\mathrm{mg} / \mathrm{mL}$ hydrocortisone (Sigma-Aldrich), 100ng/mL cholera toxin (Sigma-Aldrich), and $10 \mu \mathrm{g} / \mathrm{mL}$ insulin (Sigma-Aldrich). Ovarian carcinoma cell line OVCAR-3 (ATCC, HTB-161) was maintained in RPMI-1640 medium (Gibco) supplemented with 20\% FBS and $10 \mu \mathrm{g} / \mathrm{mL}$ insulin (Sigma Aldrich). Human epithelial carcinoma cell line A431 (ATCC, CRL-1555) was maintained in DMEM supplemented with $10 \%$ FBS. Human pharyngeal squamous cell carcinoma line FaDu (ATCC, HTB-43) was maintained in EMEM (Thermo Fisher Scientific) supplemented with 10\% FBS.

\section{Aptamers}

Nucleolin aptamer and control aptamer were synthesized by TriLink BioTechnologies (San Diego, CA). Nucleolin aptamer (NUAP) sequence [23] was $5^{\prime}$-( $\mathrm{NH}_{2}$-C6) GGT GGT GGT GGT TGT GGT GGT GGT GGT TTT TT (C6-S-S-C6)-3'. Control aptamer (CTAP) sequence: $5^{\prime}-\left(\mathrm{NH}_{2}-\mathrm{C} 6\right)$ CCT CCT CCT CCT TCT CCT CCT CCT CCT TTT TT (C6-S-S-C6)-3'.

\section{STAT3 decoy and mutant control decoy}

Sense and antisense ODN strands of STAT3 decoy and mutant decoy oligonucleotides were synthesized by GenScript (Piscataway, NJ). The STAT3 decoy sequence [7] was: $5^{\prime}-\left(\mathrm{NH}_{2}-\mathrm{C} 6\right) \mathrm{C}^{*} \mathrm{~A}^{*} \mathrm{~T}^{*}$ TTC CCG TAA ATC TTT (C6-S-S-C6)-3', 3'-( $\mathrm{NH}_{2}$-C7) $\mathrm{GT}^{*} \mathrm{~A}^{*} \mathrm{~A}^{*} \mathrm{AG}$ GGC ATT $\mathrm{T}^{*} \mathrm{~A}^{*} \mathrm{G}^{*}-5^{\prime}$ and the mutant control decoy (COTRd) sequence was: $5^{\prime}-\left(\mathrm{NH}_{2}-\mathrm{C} 6\right)$ $\mathrm{C}^{*} \mathrm{~A}^{*} \mathrm{~T}^{*}$ TTC CCT TAA ATC TTT (C6-S-S-C6)-3', $3^{\prime}-\left(\mathrm{NH}_{2}-\mathrm{C} 7\right) \mathrm{GT}^{*} \mathrm{~A}^{*} \mathrm{~A}^{*} \mathrm{AG}$ GGA ATT $\mathrm{T}^{*} \mathrm{~A}^{*} \mathrm{G}^{*}-5^{\prime}$, where * stands for phosphorothioate bond. Decoy duplexes were prepared by mixing equimolar amounts of the corresponding complementary ODNs in $25 \mathrm{mM}$ Hepes, $1 \mathrm{mM} \mathrm{MgCl}, 50 \mathrm{mM} \mathrm{NaCl}$, pH 7.4, heating the solutions to $95^{\circ} \mathrm{C}$ for $5 \mathrm{~min}$ and cooling down to room temperature. 


\section{Conjugation of STAT3 decoy strand with NHS-MAG3}

$5 \mu \mathrm{L}$ of $100 \mathrm{mM}$ solution of NHS-MAG3 in dry DMF was added to $100 \mu \mathrm{L} 500 \mu \mathrm{M}$ STAT3 decoy sense strand (STAN, has an amino group on $5^{\prime}$-end) in $0.1 \mathrm{M}$ HEPES, pH 8.0. After incubating for 30-60 min at RT, STAN-MAG3-conjugate was purified by using a P6 column (Bio-Rad) following the manufacturer's instructions. Duplex containing STAN-MAG3 was prepared as described above.

\section{Preparation of AuNP-ODNs}

The reduction of 3'-disulfide was performed by adding tris-(2-carboxyethyl)phosphine hydrochloride (Sigma-Aldrich) at a final concentration of $50 \mathrm{mM}$ followed by an incubation for $15-20 \mathrm{~min}$ at room temperature. The ODN was purified using a P6 column equilibrated with $0.1 \mathrm{M}$ MES buffer $\mathrm{pH} 6$. The thiolated ODN solution was added to $10 \mathrm{~mL}$ of $2 \mathrm{nM}$ solution of AuNP (synthesized as described before [24]) as the final molar ratio was 1:200 (AuNP:ODN) and left overnight to form the nanoconstruct (AuNP-ODN). To increase the surface concentration of aptamers on AuNP, we diluted the incubation mixture with $2.5 \mathrm{~mL}$ of $0.5 \mathrm{M} \mathrm{NaCl}$ twice, with a $4 \mathrm{~h}$ interval in between. AuNP-ODN conjugate was purified by centrifugation at $16,000 \times$ g for $15 \mathrm{~min}$ and washing the AuNP-ODN conjugate twice with $10 \mathrm{mM}$ sodium phosphate buffer, $0.1 \mathrm{M} \mathrm{NaCl}, \mathrm{pH}$ 7.0. Hydrodynamic diameters and zeta potentials of AuNP constructs were characterized using LALLS (Zetasizer Nano, Malvern Instruments Inc., Westborough, MA). The loading of ODN onto AuNP was calculated by measuring fluorescence intensities of free fluorescent ODN and the total added fluorescent ODN.

\section{Radiolabeling of MAG3-STAT3 decoy and MAG3-STAT3 decoy-AuNP}

Radiolabeling of various MAG3 conjugated preparations was performed as described [25]. Forty $\mu \mathrm{L} 0.25 \mathrm{M}$ ammonium acetate were added to 5-10 $\mu \mathrm{g}$ of MAG3-STAT3d or to 2-5 $\mu \mathrm{g}$ of AuNP conjugates followed by an addition of sodium tartrate $(50 \mathrm{mg} / \mathrm{ml})$ in sodium bicarbonate/ammonium acetate/ ammonium hydroxide buffer $(\mathrm{pH}$ 9.2). The final concentration of tartrate was $7 \mu \mathrm{g} / \mathrm{mL}$. After addition of $9.25-37 \mathrm{MBq}$ of ${ }^{99 \mathrm{~m} T c-p e r t e c h n e t a t e, ~} 2-5 \mu \mathrm{L}$ of a fresh solution of $\mathrm{SnCl}_{2} \cdot 2 \mathrm{H}_{2} \mathrm{O}(4 \mathrm{mg} / \mathrm{mL}$ in $10 \mathrm{mM}$ $\mathrm{HCl}$ ) were added. The labeling mixture was incubated at room temperature for 30-60 min. The 99mTc-labeled MAG3 AuNP was purified by gel filtration. Radiolabeling efficiency was between $70-80 \%$ before purification. After the purification radiochemical purity always exceeded $95 \%$ as determined by size-exclusion HPLC (Superdex 75, GE Healthcare Life Sciences), by instant thin-layer chromatography (ITLC) with acetone as the solvent (ITLC-SG; Gelman), or by paper chromatography (Whatman no. 1 ; VWR) with saline as mobile phase.

\section{Immunofluorescence}

Cells were fixed for $15 \mathrm{~min}$ in $4 \%$ paraformaldehyde in PBS. After several washes in PBS, the fixed cells were blocked with 5\% BSA in PBS for 1-2h. The cells were stained either with or without permeabilization with $0.1 \%$ Triton X-100/PBS using an overnight incubation with the anti-nucleolin antibody (rabbit polyclonal, ab22758, AbCam, 400x dilution with 5\% BSA/PBS). The cells were further incubated for $2 \mathrm{~h}$ with the secondary anti-rabbit $\operatorname{IgG}(\mathrm{H}+\mathrm{L})$-FITC conjugate. Cells were washed mounted in anti-fade medium (Vector Labs) containing DAPI. The slides were examined using a Leica TCS SP8 confocal microscope (Leica Microsystems, Buffalo Gove, IL) using a 63x/1.4NA lens and $95.6 \mu \mathrm{m}$ pinhole (at 1AU, $\lambda_{\mathrm{em}}=580 \mathrm{~nm}$ ) resulting in an approximate optical section thickness of $\mathrm{d}_{\mathrm{z}}=1.4 \mu \mathrm{m}$.

\section{Western blot analysis}

Heat-denatured DTT-reduced cell protein samples of A431 and FaDu cell lysate isolated by using a subcellular protein fractionation kit (Thermo Fisher Scientific) as well as HeLa whole cell lysate, positive control (Santa Cruz Biotechnology), were resolved by electrophoresis on $4-20 \%$ TGX gradient Tris/Tricine/SDS precast gels (Bio-Rad, Hercules CA). The gels were transferred to PVDF (polyvinylidene difluoride membranes, Bio-Rad), the membranes were blocked with 5\% nonfat dried milk, $0.1 \%$ Tween 20 in TBS for $2 \mathrm{~h}$ at room temperature and then incubated with anti-STAT3 (sc-482, Santa Cruz Biotechnology, Dallas, TX) or anti-nucleolin (see above) primary antibodies (diluted $1: 500$ ) at $4^{\circ} \mathrm{C}$ overnight. The bands were visualized using alkaline phosphatase- conjugated anti-rabbit antibody (A3687 Sigma-Aldrich) using BCIP/NBT (5-bromo-4-chloro3-indolyl phosphate /Nitroblue Tetrazolium) substrate solution (Roche Diagnostics). The membrane was imaged using UVP EpiChemi system.

\section{Cell Viability Assay}

Cells were plated in 96-well plates in triplicate (60-70\% confluence in $24 \mathrm{~h}$ after plating). Cells were incubated with AuNP-ODNs (20 to $100 \mathrm{nM}, 0.1 \mathrm{~mL}$ ) in Opti-MEM medium for $1 \mathrm{~d}$ at $37^{\circ} \mathrm{C} / 5 \% \mathrm{CO}_{2}$. In control wells AuNP-ODN was replaced with phosphate-buffered saline (PBS). After $1 \mathrm{~d}$ the medium was replaced with $100 \mu \mathrm{L}$ of fresh medium 
and $10 \mu \mathrm{L}$ of WST-1 reagent (Roche Molecular Biochemical) was added to each well and the plates were incubated for $0.5-3 \mathrm{~h}$ at $37^{\circ} \mathrm{C}$. The absorbance was measured using a SpectraMax M5 microplate reader (Molecular Devices) at $450 \mathrm{~nm}$. Cytotoxicity was determined by comparing the absorbance values to that of controls.

\section{ODN and AuNP-ODN constructs cell internalization study}

Cells were seeded in either 12-well plate or 96-well plates in respective media and incubated at $37^{\circ} \mathrm{C}$ with $5 \% \mathrm{CO}_{2}$. Next day the media were removed and test amounts of the aptamer (NUAP or CTAP), AuNP-ODNs, AuNP-NUAP-STAT3d or AuNPCTAP-STAT3d with fluorescent tag or radiolabeled by ${ }^{99 \mathrm{~m} T \mathrm{Tc}}$ in Opti-MEM medium were added for various time periods. After incubation, the media containing unbound fraction was removed and cells were washed twice with PBS. For dissociating the surface bound AuNP-ODN nanoconstructs, glycine buffer $(0.1 \mathrm{M}, \mathrm{pH} 2.8)$ was added and the plate incubated for $5 \mathrm{~min}$. After transferring to test tubes the cells were treated once in glycine buffer, glycine buffer fractions and PBS fractions were pooled to determine the surface-bound fraction. Following glycine buffer treatment the surface fractions were lysed with $0.2 \mathrm{M} \mathrm{NaOH} / 0.1 \%$ SDS and the lysates were counted using a Packard Gamma Counter. ODND and AuNP-ODN constructs were added to the cells at the final concentration as $0.5 \mu \mathrm{M}$. The fluorescence intensity of each lysis solution was measured by using Odyssey imager (Li-Cor Biotechnology, Lincoln NE) followed by ImageJ analysis [26].

\section{AuNP-NUAP-STAT3d cell uptake measurements}

Cells were seeded on coverslips in 12-well plates and used at $60-70 \%$ confluence. STAT3d $(0.5 \mu \mathrm{M})$ and AuNP-NUAP-STAT3d construct (5 nM AuNP with linked NUAP: $110 \mathrm{nM}$, STAT3d: $88 \mathrm{nM}$ ) with one strand of STAT3d labeled with Alexa Fluor 568 in Opti-MEM were added to the cells and incubated overnight. The cells were washed three times with PBS and fixed for $15 \mathrm{~min}$ in $4 \%$ formaldehyde. Cell plasma membrane was stained by using anti-EGFR (Cetuximab) - Alexa Fluor 488 conjugate. The cells were mounted in Vectashield anti-fading mounting medium with DAPI (Vector Laboratories, Burlingame, CA) and sealed on slides. The slides were examined using a Leica TCS SP8 confocal microscope (Leica Microsystems, Buffalo Gove, IL) using a $63 \mathrm{x} / 1.4 \mathrm{NA}$ lens and $95.6 \mu \mathrm{m}$ pinhole (at $1 \mathrm{AU}, \lambda_{\mathrm{em}}=580$ $\mathrm{nm}$ ) resulting in an approximate optical section thickness of $d_{z}=1.4 \mu \mathrm{m}$. All images were taken under the same condition. Three to four fields were imaged in three channels and saved as tiff files which were analyzed by using ImageJ software as follows: first, cell boundaries were outlined individually with ROI drawn by following green channel fluorescence signal representing anti-EGFR (Cetuximab) - Alexa Fluor 488 conjugate binding to cell membrane. The ROIs derived from the outlines of individual cells were then pasted onto the "red" channel images and the corresponding signals in the "red" (Alexa Fluor 568) channel were calculated. Fluorescence intensities were determined for 30-40 cells in three to four fields of the view and the uptake of Alexa Fluor 568-labeld STAT3d was expressed as mean intensity/cell.

\section{Cell culture radiosensitization experiments}

$\mathrm{FaDu}$ and A431 cells at $60-70 \%$ confluence in 6-well plates were treated with $20 \mu \mathrm{g} / \mathrm{ml}$ Cetuximab (Merck), STAT3d $(0.5 \mu \mathrm{M})$ and AuNP-NUAP-STAT3d nanoconstruct ( $88 \mathrm{nM}$ STAT3d, $5.2 \mathrm{nM}$ AuNP) for 20 h. After the incubation the cells were mock-irradiated or irradiated with a single dose of $4 \mathrm{~Gy}$ using a clinical linear accelerator unit (Philips, Department of Radiation Oncology, UMASS-Memorial Hospital, Worcester MA). The cells were washed with PBS twice, trypsinized, fixed and dispersed in $10 \%$ FBS/PBS. To quantify radiation therapy-induced DNA damage to cells, the cells were permeabilized and stained with FITC-labeled rabbit polyclonal antibodies against $\mathrm{Y}-\mathrm{H} 2 \mathrm{AX}$ (antiphospho-H2AX [Ser139]; Upstate Biotechnology, Lake Placid, NY) to determine the level of DNA damage in viable cells [27]. $\gamma-\mathrm{H} 2 \mathrm{AX}$ levels were measured and analyzed by flow cytometry [13, 28, 29]. Non-specific IgG-FITC conjugate was used as negative control.

\section{Statistical analysis}

$P$ values were calculated by using ordinary 2 way ANOVA analysis (alpha=0.05), Prism 7.0, GraphPad Software Inc.

\section{Results}

\section{Nucleolin aptamer and STAT3 decoy and AuNP delivery system}

Synthetic nucleolin aptamer [23] and the control aptamer (CTAP) with the guanines replaced with cytosines were used in this study; STAT3 decoy [7] was derived from the conserved hSIE genomic element found in the c-fos gene promoter and was composed of a duplex ODN with phosphorothioate modifications of the three $5^{\prime}$ - and $3^{\prime}$ - terminal nucleotides. $5^{\prime}$-end amino linker and $3^{\prime}$-end disulfide linkers were introduced to the aptamers and STAT3 sense strand. The gold surface-reactive thiol group 
reduced from a disulfide bond by DTT or TCEP was used for conjugation with AuNP (Figure 1A). As expected, AuNP cores were narrowly distributed by hydrodynamic diameter (Table 1, Figure 1B). Linker sequences composed of the three or six thymidines were inserted before the thiol group to enable the upright orientation of the ODN strand on AuNP surfaces allowing to link more aptamers or STAT3d to AuNP because the capacity of binding to the target may be compromised otherwise [30]. To ensure that fluorescence of NUAP/STAT3d-AuNP is only minimally affected by gold surface plasmons and is strong enough for cell imaging, amino group on the 5 -end of ODNs was used for conjugating with Alexa Fluor 488, Alexa Fluor 568 (for confocal microscopy) or Cy5.5 and 800CW (for NIR imaging) (Figure 1A). Amino group was also used to conjugate ODNs with MAG3 ligand suitable for radiolabeling with reduced [99mTc] pertechnetate in the cell internalization study. All six engineered AuNP-NUAP/STAT3d nanoconstructs (Table 1 and Table S1) were stable in the presence inorganic anions including phosphate and could be stored for months at $4^{\circ} \mathrm{C}$. The formation of aptamer- and STAT3d - linked AuNP-ODNs nanoconstructs was analyzed by using non-denaturating gel electrophoresis which demonstrated the presence of large (non-migrating) dual-fluorescence labeled species indicating co-localization of the aptamer and the duplex on the same AuNP (Figure 1C). The total amount (aptamer or STAT3 decoy) bound on AuNP was determined by calculating the fluorescence intensity difference between the added ODN and free ODN in supernatant after AuNP-ODNs purification by centrifugation. The amount of ODN (aptamer or STAT3 decoy) bound to AuNP varied by the sequence and length with shorter ODN having an advantage of higher relative content of phosphorothioates in the ODNs. AuNP-NUAP had the lowest yield of binding to AuNP and resulted in AuNP aggregation because NUAP contains a plurality of guanines in the sequence. When NUAP and STAT3d were added together to AuNP, the yield AuNP-NUAP-STAT3d was higher than AuNP-NUAP synthesized by adding NUAP only. Hydrodynamic radii and other characteristics of AuNP and AuNP-ODN nanoconstructs are shown in Table 1.

Table 1. Characterization of gold nanoparticle-based constructs used in this study.

\begin{tabular}{|c|c|c|c|c|c|}
\hline Name & Diameter by number, $\mathrm{nm}^{\mathrm{b}}$ & Zeta potential, $\mathrm{mV}$ & $\begin{array}{l}\text { AuNP core diameter by } \\
\text { TEM, nm }\end{array}$ & $\begin{array}{l}\text { ODN bound to AuNP (\% of } \\
\text { added) }{ }^{c}\end{array}$ & $\begin{array}{l}\text { ODN/AuNP ratio } \\
\mathrm{mol} / \text { particle }\end{array}$ \\
\hline $\mathrm{AuNPa}$ & 11.9 & -21.7 & $13.5 \pm 1.5$ & - & - \\
\hline AuNP-NUAP & & - & - & 2.5 & 224 \\
\hline AuNP-CTAP & 71.4 & -26.8 & & 10.3 & 84 \\
\hline AuNP-STAT3d & 2.9 & -12.9 & & 46.1 & 101 \\
\hline AuNP-COTRd & 49.4 & -28.7 & & 27.7 & 74 \\
\hline AuNP-NUAP-STAT3d & 18.2 & -26.8 & $17.3 \pm 1.4$ & 8.2 & $\begin{array}{l}\text { NUAP: } 49 \\
\text { STAT3d: } 60\end{array}$ \\
\hline AuNP-CTAP-STAT3d & 52.4 & -29.5 & & 16.3 & $\begin{array}{l}\text { CTAP: } 38 \\
\text { STAT3d: } 60\end{array}$ \\
\hline
\end{tabular}

a) AuNP were synthesized as described in [24]; b) hydrodynamic diameters (number average); c) a ratio of [bound ODN]/ [added ODN]*100.
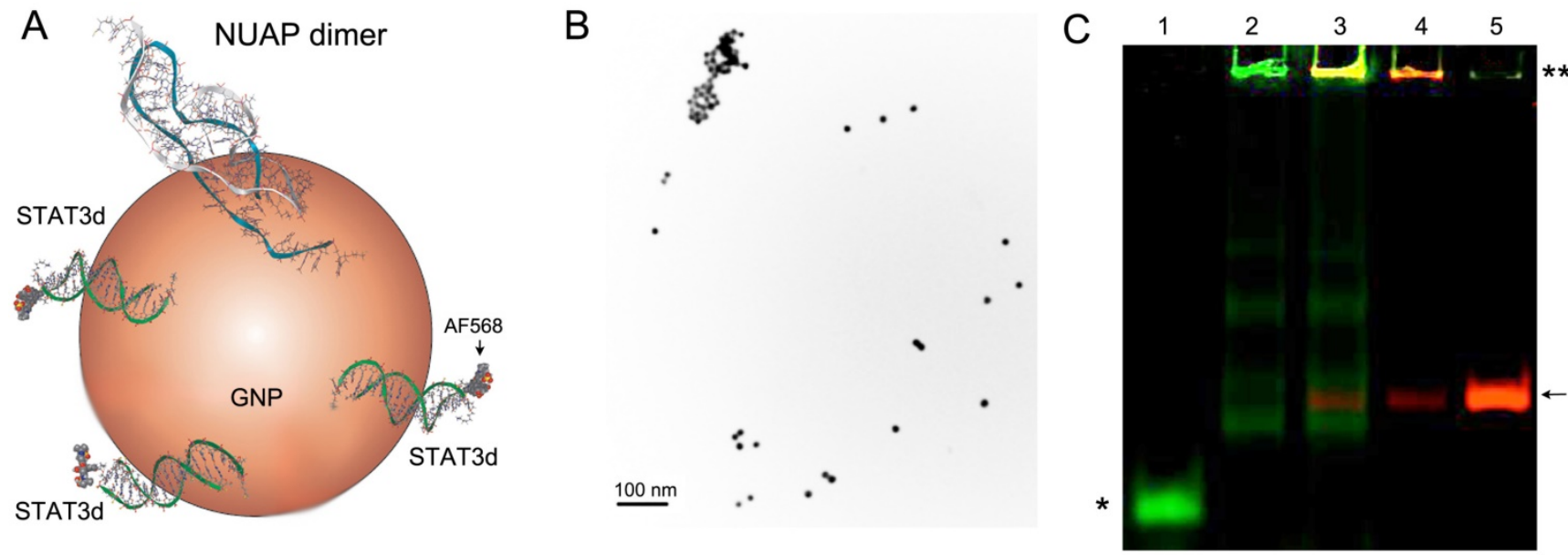

Figure 1. A - a scheme showing a gold nanoparticle with cell-surface specific nucleolin aptamer (NUAP) that forms a quadruplex dimer and Alexa Fluor 568 labeled STAT3-binding duplex (STAT3d); B - transmission electron microscopy of AuNPs, bar = $100 \mathrm{~nm}$; C- a pseudo-color fluorescent image of a polyacrylamide gel (10\% TBE) showing electrophoretic analysis of AuNP-ODN constructs and their components, lane: 1) STAT3d; 2) AuNP-STAT3d; 3) AuNP-CTAP-STAT3d; 4) AuNP-CTAP; 5) CTAP. Control aptamer CTAP was labeled with Cy5.5 (red) and one of the strands of STAT3d was labeled with NIR Dye 800CW (green). An asterisk shows the position of STAT3d-800CW, double asterisk- AuNP conjugates retained at the start; arrow- NUAP-Cy5.5 


\section{Expression of nucleolin on cell surface of HNC cells and STAT3 overexpression}

Because overexpression of nucleolin on HNC $\mathrm{FaDu}$ cell surface is crucial for cellular uptake and trafficking of AuNP-ODN constructs in this study, we first tested the levels of nucleolin expression on the surface. This was performed by using immunofluorescence due to additional expression of nucleolin in cytoplasmic and nuclear fraction of cells. Human epithelial carcinoma A431 cells were used as positive control because they express large amounts of nucleolin on the cell surface. NIH-OVCAR3 cell line, an ovarian carcinoma cell line with low expression of surface nucleolin was used as negative control. The total level of nucleolin expression by immunofluorescence in the HNC FaDu cell line was similar to that of A431 and NIH-OVCAR3 cells (Figure 2). However, in both HNC lines nucleolin was present in the cytoplasm while in OVCAR cells nucleolin was detectable mostly in the nucleoli (Figure 2A-C). After subcellular fractionation of $\mathrm{FaDu}$ and A431 we determined that nucleolin was present in the membrane fraction including plasma membrane, which was confirmed by Western blotting (Figure 2F). We detected both full-length nucleolin $(106 \mathrm{kDa})$ and the products of proteolysis [31] in the fractions of all cells we tested. Furthermore, we tested whether HNC FaDu cells express functional STAT3 by detecting STAT3 protein by EMSA (Figure 2E) and on Western blots (Figure 2F). EMSA showed the ability of STAT3d to bind to the protein product present in cell lysates after STAT3d was assembled from one or two ODNs carrying fluorescent dyes. We also found that in protein lysates of FaDu cells the levels of expression of STAT3 were similar to the levels detected in the positive control, i.e. in HeLa cells extract, Figure 2F.
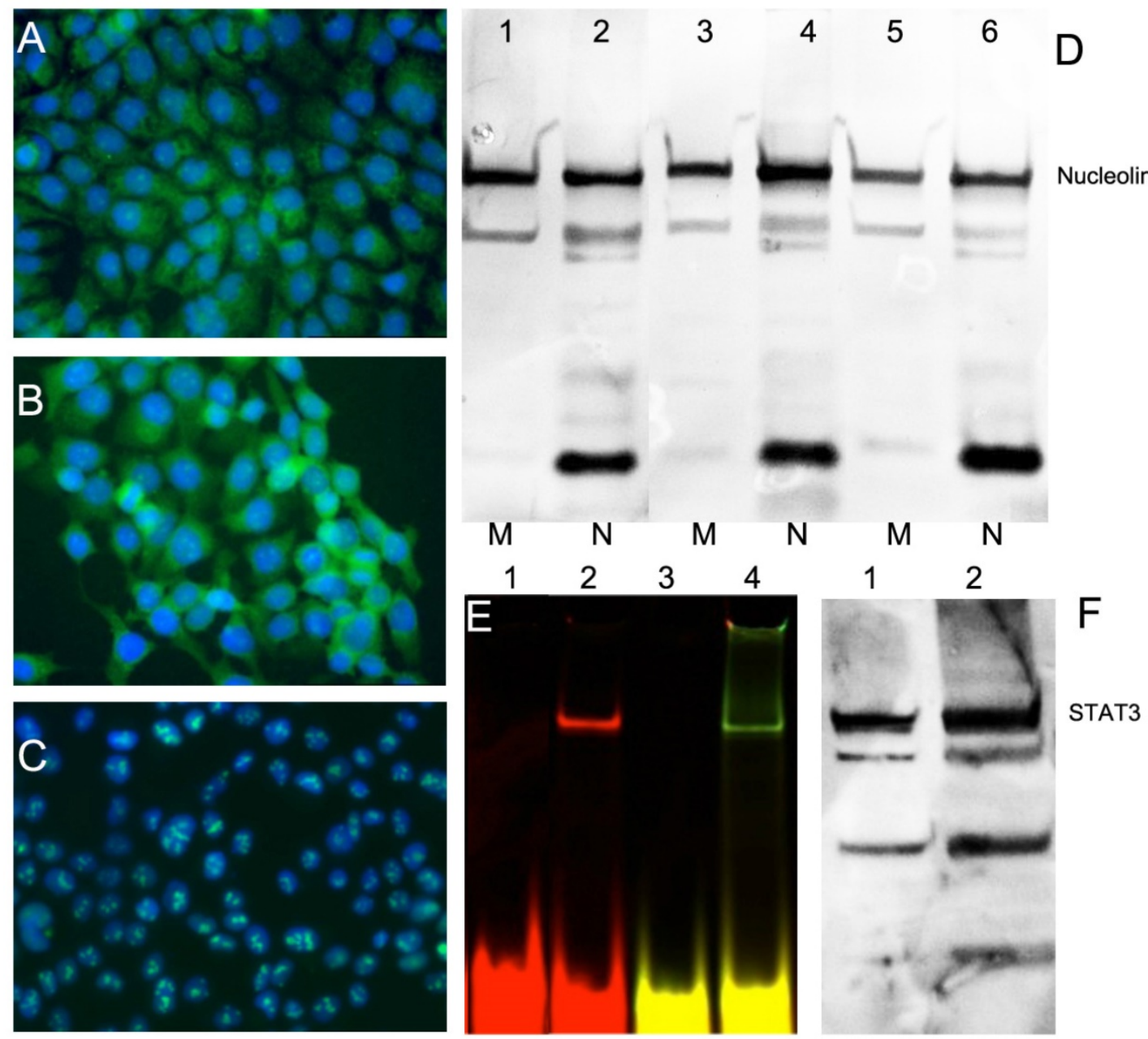

Nucleolin

Figure 2. Expression of nucleolin (cell-surface target protein) and STAT3 in epithelial cancer cell lines. Immunofluorescence using anti-nucleolin antibody showing nucleolin expression in epithelioid carcinoma A431 (A), HNC line FaDu (B); ovarian carcinoma OVCAR3 (C). Secondary FITC-conjugated IgG (green) was used. Nuclei were counterstained with DAPI (blue). D- nucleolin expression in membrane (M) and nuclear (N) fractions of cells. Lanes 1,2 - A431, Lanes 3,4 - FaDu, lanes 5,6- HeLa cells. E- electrophoretic mobility shift assay (EMSA) showing STAT3d alone (lane 1,3) and after adding FaDu cell lysate (lane 2,4). STAT3 d was labeled either using Cy5.5 (lanes 1,2) or dual-labeled with Cy5.5 and 800CW(lanes3, 4); F- Western blotting of STAT3 in FaDu cell extract (lane 1) and HeLa extract (lane 2, positive control). 


\section{Cytotoxicity of AuNP-ODN nanoconstructs}

Cytotoxicity tests of all synthesized AuNP conjugates with ODNs (AuNP-NUAP, AuNP-CTAP, AuNP-STAT3d and AuNP-COTRd) were performed in $\mathrm{FaDu}$ and control non-HNC cells at various concentrations. We observed no measureable cytotoxicity below $0.1 \mu \mathrm{M}$ with no significant differences between these AuNP constructs and $1 \mu \mathrm{M}$ for aptamers and STAT3 decoys.

\section{Cell uptake of STAT3d, nucleolin aptamer and corresponding AuNP nanoconstructs}

To compare the cell uptake of various AuNP nanoconstructs and free aptamers, we conjugated NIR dyes $800 \mathrm{CW}$ and Cy5.5 to aptamers and STAT3d, respectively, which enabled very high sensitivity of detection. We investigated whether there were measurable differences in the uptake of nanoconstructs carrying either nucleolin aptamer NUAP or the control aptamer CTAP at four different concentrations in cells. Initially we observed that independently of cell line free NUAP aptamer showed higher binding to the cells than free CTAP (Figure 3A). By using the same fluorescent detection method we then established that Cy5.5- labeled AuNP-NUAP constructs showed about 4-times higher cell uptake than AuNP-CTAP constructs in both $\mathrm{FaDu}$ and A431 cells. By performing cell fractionation and measuring fluorescence intensity of cell surface and cell-internalized fractions separately we determined that on average, more than $90 \%$ of the AuNP constructs were internalized (Figure 3B). We further performed a quantitative comparative uptake assay of STAT3d, AuNP-STAT3d and AuNP-NUAP-STAT3d which carried one strand of
STAT3d radiolabeled with [99mTc] (Figure 3C). Linking STAT3d to AuNP surface resulted in a strong increase of cell uptake of STAT3d: after conjugating with AuNP and with nucleolin aptamer-bound AuNP, STAT3d uptake increased from $0.12 \pm 0.01 \%$ to $3.5 \pm 0.51 \%$ in A431 cells resulting in approximately a 30 -times increase and from $0.18 \pm 0.01 \%$ to $1.87 \pm 0.27 \%$ in $\mathrm{FaDu}$ cells, i.e. a 10 -fold increase. To visualize the uptake of AuNP nanoconstructs with confocal microscopy, we pre-labeled ODNs with Alexa Fluor 568 fluorophore before conjugating to the surface of AuNP. Figure 4 shows the localization of the AuNP-NUAP-STAT3d in the cytoplasm and around DAPI-stained nuclei (blue channel) in FaDu and A431 cell lines. The evidence of the uptake observed by cell microscopy was consistent with the results of cell lysate NIR fluorescence analysis and measurements of radioactivity (Figure 4). The internalization of STAT3 decoy in cell nuclei as well as in the cytoplasm was clearly visible in many A431 and FaDu cells in the monolayer culture while no evidence of the uptake was observed in the case of free STAT3d. Since we additionally stained plasma membrane by using anti-EGFR (Cetuximab)- Alexa Fluor 488 conjugate, we were able to quantify the Alexa Fluor 568-labeled STAT3d in each cell individually. Quantitative analysis of optical section volume average intensities (expressed in arbitrary units of fluorescence, AU) showed that A431 cells were internalizing NUAP-targeted AuNP-STAT3d construct more efficiently than STAT3d (35.9 AU AuNP-NUAP-STAT3d vs. 12.1 AU STAT3d). The same trend was observed in the case of FaDu cells: 30.5 AU AuNP-NUAP-STAT3d vs. 10.4 AU STAT3d (Figure 4).
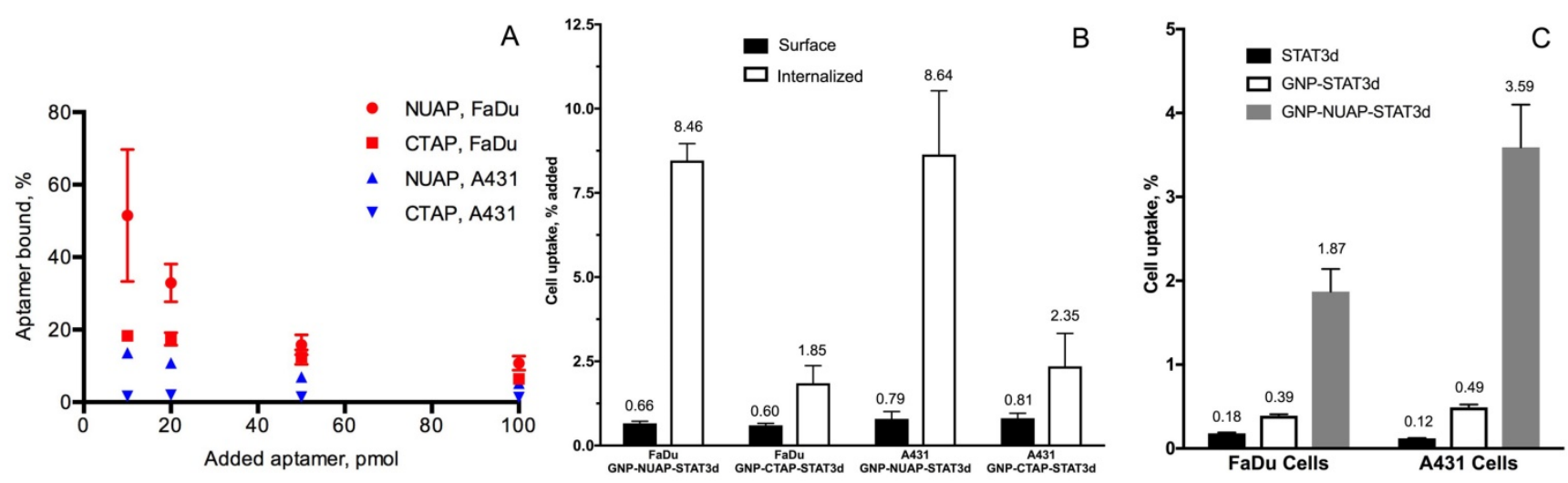

Figure 3. The uptake of aptamers and aptamer-linked AuNP nanoconstructs in cell culture. A- Total cell uptake of Cy5.5-labeled nucleolin-specific aptamer (NUAP) and control non-specific aptamer (CTAP) incubated with HNC cell line FaDu and squamous carcinoma A431 for $4 \mathrm{~h}$. The uptake was determined by using measurements of Cy5.5 fluorescence in cell lysates; B- fractional content of surface-bound and internalized AuNP-aptamer-STAT3d constructs in FaDu and A431 cells. The \% of total in the bound and internalized fractions was determined by measuring fluorescence of cell lysates after fractionation (surface and internalized). AuNP constructs were incubated in the cell culture for $4 \mathrm{~h}$ at $37{ }^{\circ} \mathrm{C}$. C- Internalization of STAT3d, AuNP-STAT3d and AuNP-NUAP-STAT3d in FaDu and A431 cells by $[99 \mathrm{mTc}]$ radiolabeling of a one of the strand of STAT3d by using modification with MAG3 chelate. 

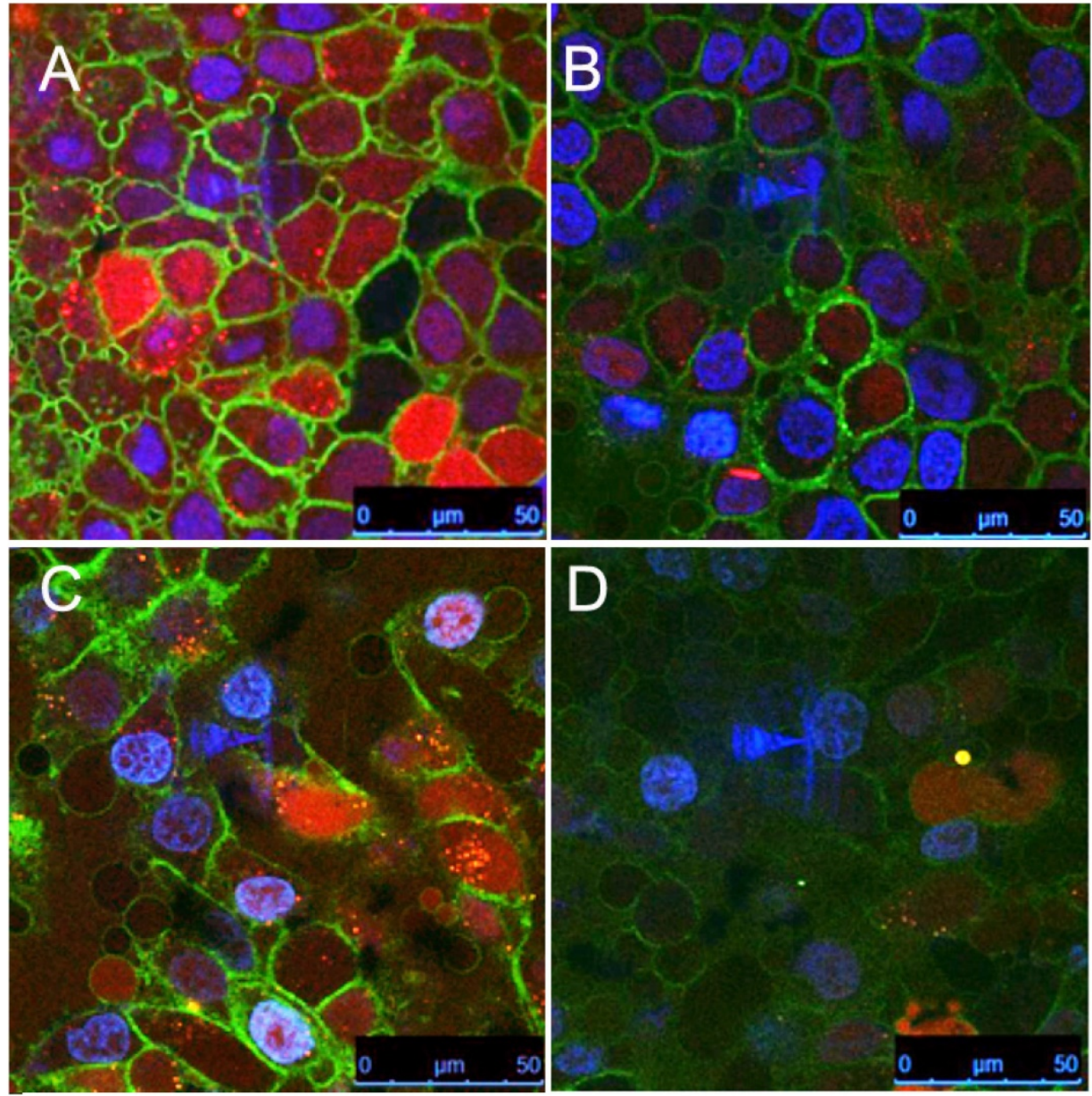

Figure 4. Confocal multichannel microscopy of STAT3d and AuNP-NUAP-STAT3d uptake in epithelial cancer cells after incubating with A431 cell culture (A, B) or FaDu cells (C, D); AuNP-NUAP-STAT3d (A - A431 cells; C - FaDu cells); STAT3 duplex (B - A431 cells; D - FaDu cells). Blue- DAPI; Green - anti-EGFR-Alexa Fluor488 for staining cell plasma membrane; Red - Alexa Fluor 568 (one strand of STAT3 duplex).

\section{Potentiation of STAT3d radiosensitization effect}

To investigate whether AuNP-NUAP-STAT3d constructs exhibited radiosensitization effect in A431 and $\mathrm{FaDu}$ cells, the cells were subjected to a single-dose radiation treatment (RT) in the presence or in the absence of STAT3d and AuNP-NUAP-STAT3d nanoconstructs after 20h incubation. Cetuximab was used as "standard of care" therapeutic humanized antibody for head and neck cancer treatment. Both viability and the extent of genomic DNA damage was assessed by flow cytometry. This experiment showed that A431 cells were more radiosensitive after treating with experimental AuNP-NUAP-STAT3d or STAT3d than FaDu cells. The percent of viable A431 cell numbers decreased by $20 \%$ in STAT3d and AuNP-NUAP-STAT3d groups compared to the control cells. The degree of cell DNA damage caused by constructs showed the following trend in A431 cells: Cetuximab>AuNP-NUAP-STAT3d $>$ STAT3d.
Unlike A431, FaDu cells showed high level of radioresistance, $\mathrm{FaDu}$ cell viability did not change after RT was delivered. Among live cell fraction, DNA damage measurement (Figure 5B) showed that AuNP-NUAP-STAT3d construct, STAT3d and Cetuximab caused DNA damage to the cells after $20 \mathrm{~h}$ incubation. The cell DNA damage levels did not change significantly before and after RT for Cetuximab group. However, cell DNA damage increased almost by $50 \%$ after RT in the case of AuNP-NU-STAT3d and STAT3d groups. Inhibition of STAT3 resulted in radiosensitization of HNC FaDu cells. Unlike Cetuximab, STAT3d and AuNP-NUAP-STAT3d showed a small but statistically significant increase of DNA damage in RT-treated FaDu cells $(\mathrm{p}<0.005)$. While STAT3d and AuNP-NUAP-STAT3d showed similar radiosensitization effects in FaDu cells, the actual STAT3d concentrations for achieving this effect were different with free STAT3d $(500 \mathrm{nM})$ being 4-5 times higher than AuNP-NUAP-STAT3d (88 nM STAT3d). 


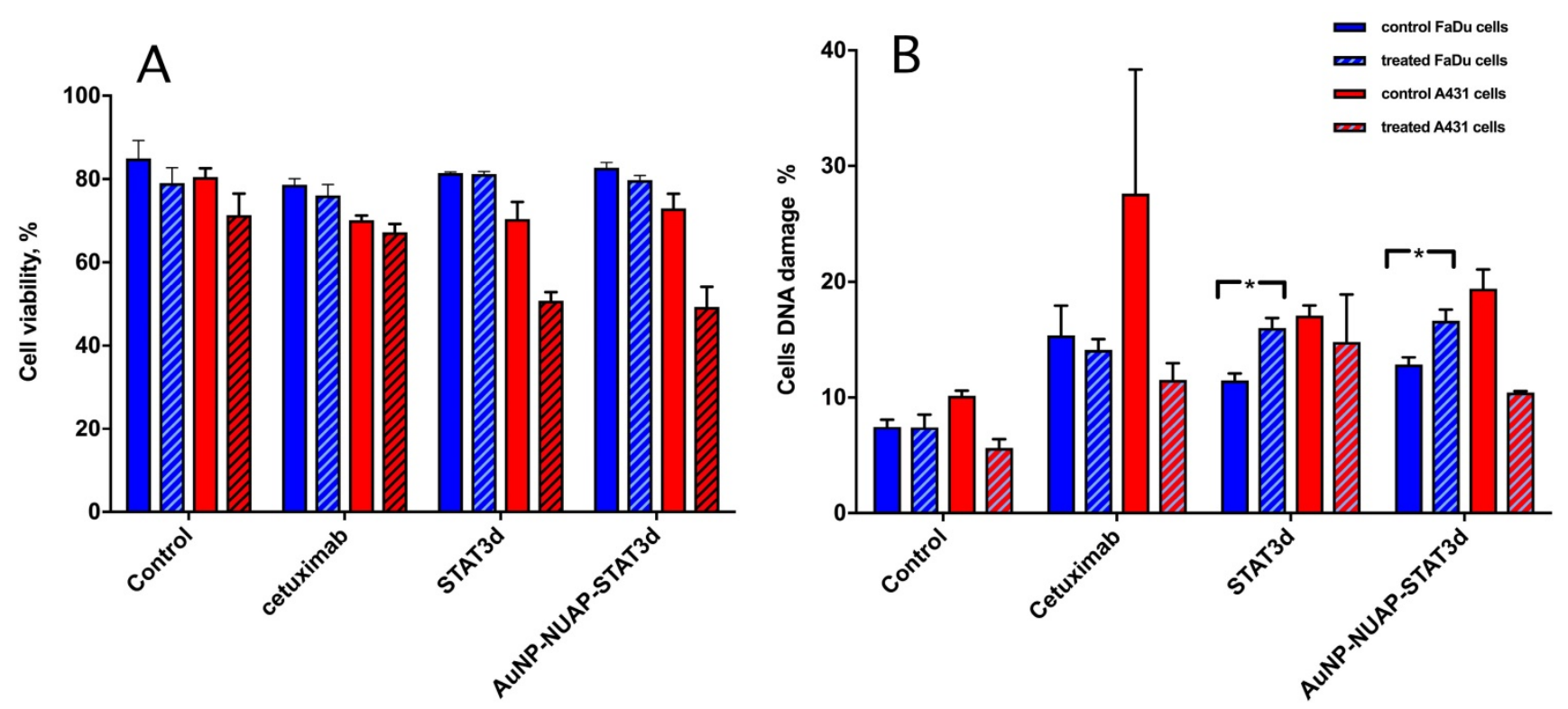

Figure 5. The effect of RT on viability (A) of A431 (blue bars) and FaDu cells (red bars) and genomic DNA damage (B) pre- (solid bars) and post- (hatched bars) radiation treatment. Both viability and the damage to DNA were determined by using flow cytometry. Control cells (no pre-incubation), or experimental cells incubated with 1) Cetuximab (control as a standard immunotherapy for head and neck cancer), 2) STAT3d or 3) AuNP-NUAP-STAT3d for 20h and then either mock-irradiated or irradiated with a single dose of 4Gy. Staining performed with FITC- labeled rabbit polyclonal anti- $y$-H2AX antibodies and analyzed by FACS. * Indicates statistical significance, $\mathrm{p}<0.005$.

\section{Discussion}

Human head and neck cancers exhibit highly malignant phenotypes characterized by the extensive invasion into surrounding tissues and metastasis to distant organs, even at an early stage $[32,33]$. Recent studies have identified several promising therapeutic targets in HNC [34]. Of these, STAT3 is a leading candidate given its increased expression in $\mathrm{HNC}$, which promotes tumor metastasis, angiogenesis, immune evasion and suppression [35-37]. STAT3 is being explored in both pre-clinical models and in early clinical trials [38] because of its proven role in the pathogenesis and progression of malignant tumors $[3,5,39]$. STAT3 decoy oligonucleotide duplex specifically binds to activated STAT3 and blocks binding of STAT3 to DNA sequences on STAT3-responsive promoters, resulting in the inhibition of STAT3-mediated transcription, potentially inhibiting tumor cell proliferation $[2,40]$. Since phosphorodiester ODNs are unstable, STAT3d must be delivered via local or intratumoral injection. To overcome this drawback, phosphorothiates were introduced to both termini of STAT3d backbone [41, 42]. As a consequence, the stability of STAT3d increased resulting in extended blood half-life [43-45]. AuNPs are also potentially able to increase the stability of STAT3d and are capable of high capacity delivery of STAT3d into the cells. To increase the delivery of nucleolin aptamer or other ODNs such as aptamers or siRNA they were linked to AuNP or loaded into liposomes. To improve the uptake of AuNP nanoconstructs in HNC, we chose to explore cell-surface nucleolin aptamer targeting by using an AuNP-based delivery system. It has been reported that nucleolin aptamer completely inhibited the proliferation and exhibited cytotoxicity towards MCF-7 cells [46, 47]. Nucleolin aptamer, a G-quadruplex oligonucleotide, was shown to have high binding affinity to nucleolin with $\mathrm{Kd}$ ranging from $\mathrm{pM}$ to low $\mathrm{nM}$ range [48]. It has favorable pharmacokinetic properties and a high safety profile due to small size, and the absence of immunogenicity [23, 46, 49]. Therefore we synthesized AuNP-NUAP-STAT3d nanoconstructs with STAT3d cargo and nucleolin aptamer appended to the AuNP surface to further enhance cancer cell-specific uptake of STAT3d under the effect of nucleolin aptamer and AuNP. In HNC treatment radiation therapy is typically combined with surgical approaches either as post-operative adjuvant radiotherapy or as primary option in treating laryngeal tumors for organ preservation [50]. Since AuNPs are potent radiosensitizers [51- 53], AuNP-NUAP-STAT3d has the potential to serve as radiosensitizer but the ability of nanoconstructs to internalize in cells had to be tested. While cytoplasmic localization of AuNP is sufficient to yield radiosensitization, nuclear penetration enables additional DNA damage via physical dose enhancement by the short-range low energy electrons (LEEs) and chemical enhancement by the very LEEs. The internalization of 
AuNP-NUAP-STAT3d was critical for our study and A431 and FaDu cells were good candidates for internalization studies since both cell lines express nucleolin on cell surface. By using site-specific [ ${ }^{99 \mathrm{mTc}}$ ]labeling of ODN strands with radioisotopes and fluorescence intensity measurements we showed that AuNP-NUAP-STAT3d is capable to internalize in HNC FaDu cells. Nucleolin aptamer of AuNP nanoconstruct assisted in the internalization of AuNP-NUAP-STAT3d. This result is consistent with published data that showed dense packing of NUAP on AuNP favoring the internalization of NUAP-AuNP in a wide range of cancer cells [54]. In our study, AuNP-NUAP-STAT3d were able to deliver almost 30 times more STAT3d than STAT3d alone to A431 cells and 10 times more into FaDu cells. It is important to note that it is more practical to utilize NUAP as a cell-specific targeting agent rather than therapeutic agent because the required quantity of NUAP is much lower. It has been previously established that STAT3 inhibition resulted in potential increase of radiosensitivity in HNC either by using small molecule inhibitors, small interfering RNA (siRNA) or short hairpin RNA (shRNA) [36, 37, 55, 56]. Our results suggest that radiosensitization effect of STAT3d can also be enhanced by gold nanoparticles, which likely induce apoptosis via enhanced DNA double-strand break formation.

In conclusion, we demonstrated that 1) both STAT3d duplex and STAT3d-linked to nucleolin-targeted AuNPs nanoconstructs show similar decrease of viability in A431 cells (20\%) after receiving irradiation, 2) they result in radiosensitization effect in radioresistant $\mathrm{FaDu}$ cells. The linking of STAT3d duplex to AuNP-NUAP resulted in a more efficient STAT3d internalization in cells than STAT3d alone. The improved internalization of AuNP-NUAP nanoconstructs and the presence of AuNP cores were both contributing to the amplification of STAT3d effect in FaDu cells which was observed at a 4-times lower concentration of STAT3d (as a component of AuNP-NUAP-STAT3d, $88 \mathrm{nM})$ than in the case of STAT3d alone $(500 \mathrm{nM})$.

\section{Acknowledgment}

This work was supported in part by grants from the NIH: R21EB017980 (SZ), RO1 EB000858 and RO1 DK095728 (AB). We thank staff of Department of Radiation Oncology, UMASS-Memorial Hospital for performing cell irradiation study and Dr. Gang Han for providing the access to Malvern ZetaSizer instrument.

\section{Supplementary Material}

Table S1. http:/ / www.ntno.org/v02p0001s1.pdf

\section{Competing Interests}

The authors have declared that no competing interest exists.

\section{References}

1. Siegel RL, Miller KD, Jemal A. Cancer Statistics, 2017. CA Cancer J Clin. 2017; 67: 7-30

2. Sen M, Thomas SM, Kim S, Yeh JI, Ferris RL, Johnson JT, et al. First-in-human trial of a STAT3 decoy oligonucleotide in head and neck tumors: implications for cancer therapy. Cancer Discov. 2012; 2: 694-705

3. Grandis JR, Drenning SD, Chakraborty A, Zhou MY, Zeng Q, Pitt AS, et al. Requirement of Stat3 but not Stat1 activation for epidermal growth factor receptor- mediated cell growth In vitro. J Clin Invest. 1998; 102: 1385-92.

4. Pedranzini L, Leitch A, Bromberg J. Stat3 is required for the development of skin cancer. J Clin Invest. 2004; 114: 619-22.

5. Grandis JR, Drenning SD, Zeng Q, Watkins SC, Melhem MF, Endo S, et al Constitutive activation of Stat 3 signaling abrogates apoptosis in squamous cell carcinogenesis in vivo. Proc Natl Acad Sci U S A. 2000; 97: 4227-32.

6. Spitzner M, Ebner R, Wolff HA, Ghadimi BM, Wienands J, Grade M. STAT3: A Novel Molecular Mediator of Resistance to Chemoradiotherapy. Cancers (Basel). 2014; 6: 1986-2011.

7. Leong PL, Andrews GA, Johnson DE, Dyer KF, Xi S, Mai JC, et al. Targeted inhibition of Stat3 with a decoy oligonucleotide abrogates head and neck cancer cell growth. Proc Natl Acad Sci U S A. 2003; 100: 4138-43.

8. Xi S, Gooding WE, Grandis JR. In vivo antitumor efficacy of STAT3 blockade using a transcription factor decoy approach: implications for cancer therapy. Oncogene. 2005; 24: 970-9.

9. Mirkin CA, Letsinger RL, Mucic RC, Storhoff JJ. A DNA-based method for rationally assembling nanoparticles into macroscopic materials. Nature. 1996; 382: 607-9.

10. Dhar S, Daniel WL, Giljohann DA, Mirkin CA, Lippard SJ. Polyvalent oligonucleotide gold nanoparticle conjugates as delivery vehicles for platinum(IV) warheads. J Am Chem Soc. 2009; 131: 14652-3.

11. Prigodich AE, Randeria PS, Briley WE, Kim NJ, Daniel WL, Giljohann DA, et al. Multiplexed nanoflares: mRNA detection in live cells. Anal Chem. 2012; 84: 2062-6.

12. Zhang XD, Wu D, Shen X, Chen J, Sun YM, Liu PX, et al. Size-dependent radiosensitization of PEG-coated gold nanoparticles for cancer radiation therapy. Biomaterials. 2012; 33: 6408-19.

13. Zhang P, Qiao Y, Xia J, Guan J, Ma L, Su M. Enhanced radiation therapy with multilayer microdisks containing radiosensitizing gold nanoparticles. ACS Appl Mater Interfaces. 2015; 7: 4518-24.

14. Ma N, Jiang $\mathrm{YW}$, Zhang $\mathrm{X}, \mathrm{Wu} \mathrm{H}$, Myers JN, Liu $\mathrm{P}$, et al. Enhanced Radiosensitization of Gold Nanospikes via Hyperthermia in Combined Cancer Radiation and Photothermal Therapy. ACS Appl Mater Interfaces. 2016

15. Ma N, Wu FG, Zhang X, Jiang YW, Jia HR, Wang HY, et al. Shape-Dependent Radiosensitization Effect of Gold Nanostructures in Cancer Radiotherapy: Comparison of Gold Nanoparticles, Nanospikes, and Nanorods. ACS Appl Mater Interfaces. 2017: 9: 13037-48.

16. Ma N, Liu P, He N, Gu N, Wu FG, Chen Z. Action of Gold Nanospikes-Based Nanoradiosensitizers: Cellular Internalization, Radiotherapy, and Autophagy. ACS Appl Mater Interfaces. 2017.

17. Liu L, Zheng M, Renette T, Kissel T. Modular Synthesis of Folate Conjugated Ternary Copolymers: Polyethylenimine-graft-Polycaprolactone-block-Poly(ethylene glycol)-Folate for Targeted Gene Delivery. Bioconjug Chem. 2012

18. Kim E, Yang J, Park J, Kim S, Kim NH, Yook JI, et al. Consecutive Targetable Smart Nanoprobe for Molecular Recognition of Cytoplasmic microRNA in Metastatic Breast Cancer. ACS Nano. 2012.

19. Bates PJ, Choi EW, Nayak LV. G-rich oligonucleotides for cancer treatment. Methods Mol Biol. 2009; 542: 379-92.

20. Reyes-Reyes EM, Teng Y, Bates PJ. A new paradigm for aptamer therapeutic AS1411 action: uptake by macropinocytosis and its stimulation by a nucleolin-dependent mechanism. Cancer Res. 2010; 70: 8617-29.

21. Bates PJ, Kahlon JB, Thomas SD, Trent JO, Miller DM. Antiproliferative activity of G-rich oligonucleotides correlates with protein binding. J Biol Chem. 1999; 274: 26369-77.

22. Ginisty H, Sicard H, Roger B, Bouvet P. Structure and functions of nucleolin. J Cell Sci. 1999; 112 ( Pt 6): 761-72.

23. Teng Y, Girvan AC, Casson LK, Pierce WM, Jr., Qian M, Thomas SD, et al. AS1411 alters the localization of a complex containing protein arginine methyltransferase 5 and nucleolin. Cancer Res. 2007; 67: 10491-500.

24. Bogdanov AA, Jr., Gupta S, Koshkina N, Corr SJ, Zhang S, Curley SA, et al. Gold nanoparticles stabilized with MPEG-grafted poly(l-lysine): in vitro and in vivo evaluation of a potential theranostic agent. Bioconjug Chem. 2015; 26: 39-50.

25. Hnatowich DJ, Winnard P, Jr., Virzi F, Fogarasi M, Sano T, Smith CL, et al. Technetium-99m labeling of DNA oligonucleotides. J Nucl Med. 1995; 36: 2306-14. 
26. Schneider CA, Rasband WS, Eliceiri KW. NIH Image to ImageJ: 25 years of image analysis. Nat Methods. 2012; 9: 671-5.

27. Kuo LJ, Yang LX. Gamma-H2AX - a novel biomarker for DNA double-strand breaks. In Vivo. 2008; 22: 305-9.

28. Huang X, Darzynkiewicz Z. Cytometric assessment of histone H2AX phosphorylation: a reporter of DNA damage. Methods Mol Biol. 2006; 314: 73-80.

29. Hou YN, Lavaf A, Huang D, Peters S, Huq R, Friedrich V, et al. Development of an automated gamma-H2AX immunocytochemistry assay. Radiat Res. 2009; 171: 360-7.

30. Storhoff JJ, Elghanian R, Mirkin CA, Letsinger RL. Sequence-Dependent Stability of DNA-Modified Gold Nanoparticles. Langmuir. 2002; 18: 6666-70.

31. Chen CM, Chiang SY, Yeh NH. Increased stability of nucleolin in proliferating cells by inhibition of its self-cleaving activity. J Biol Chem. 1991; 266: 7754-8.

32. Koontongkaew $\mathrm{S}$. The tumor microenvironment contribution to development, growth, invasion and metastasis of head and neck squamous cell carcinomas. J Cancer. 2013; 4: 66-83.

33. Pulte $\mathrm{D}$, Brenner $\mathrm{H}$. Changes in survival in head and neck cancers in the late 20th and early 21st century: a period analysis. Oncologist. 2010; 15: 994-1001.

34. Ferreira MB, De Souza JA, Cohen EE. Role of molecular markers in the management of head and neck cancers. Curr Opin Oncol. 2011; 23: 259-64.

35. Leeman RJ, Lui VW, Grandis JR. STAT3 as a therapeutic target in head and neck cancer. Expert opinion on biological therapy. 2006; 6: 231-41.

36. Chen CC, Chen WC, Lu CH, Wang WH, Lin PY, Lee KD, et al. Significance of interleukin-6 signaling in the resistance of pharyngeal cancer to irradiation and the epidermal growth factor receptor inhibitor. Int J Radiat Oncol Biol Phys. 2010; 76: 1214-24.

37. Li X, Wang H, Lu X, Di B. Silencing STAT3 with short hairpin RNA enhances radiosensitivity of human laryngeal squamous cell carcinoma xenografts in vivo. Exp Ther Med. 2010; 1: 947-53.

38. Hammerman PS, Hayes DN, Grandis JR. Therapeutic insights from genomic studies of head and neck squamous cell carcinomas. Cancer Discov. 2015; 5: 239-44.

39. Masuda M, Suzui M, Yasumatu R, Nakashima T, Kuratomi Y, Azuma K, et al. Constitutive activation of signal transducers and activators of transcription 3 correlates with cyclin D1 overexpression and may provide a novel prognostic marker in head and neck squamous cell carcinoma. Cancer Res. 2002; 62: 3351-5.

40. Shen J, Li R, Li G. Inhibitory effects of decoy-ODN targeting activated STAT3 on human glioma growth in vivo. In Vivo. 2009; 23: 237-43.

41. Stein CA, Subasinghe C, Shinozuka K, Cohen JS. Physicochemical properties of phosphorothioate oligodeoxynucleotides. Nucleic Acids Res. 1988; 16: 3209-21.

42. Marshall WS, Caruthers MH. Phosphorodithioate DNA as a potential therapeutic drug. Science. 1993; 259: 1564-70.

43. Dam DH, Culver KS, Odom TW. Grafting aptamers onto gold nanostars increases in vitro efficacy in a wide range of cancer cell types. Mol Pharm. 2014; 11: 580-7.

44. Xue J, Shan L, Chen H, Li Y, Zhu H, Deng D, et al. Visual detection of STAT5B gene expression in living cell using the hairpin DNA modified gold nanoparticle beacon. Biosens Bioelectron. 2013; 41: 71-7.

45. Cao Z, Tong R, Mishra A, Xu W, Wong GC, Cheng J, et al. Reversible cell-specific drug delivery with aptamer-functionalized liposomes. Angew Chem Int Ed Engl. 2009; 48: 6494-8.

46. Soundararajan S, Chen W, Spicer EK, Courtenay-Luck N, Fernandes DJ. The nucleolin targeting aptamer AS1411 destabilizes Bcl-2 messenger RNA in human breast cancer cells. Cancer Res. 2008; 68: 2358-65.

47. Malik MT, O'Toole MG, Casson LK, Thomas SD, Bardi GT, Reyes-Reyes EM, et al. AS1411-conjugated gold nanospheres and their potential for breast cancer therapy. Oncotarget. 2015; 6: 22270-81.

48. Bates PJ, Laber DA, Miller DM, Thomas SD, Trent JO. Discovery and development of the G-rich oligonucleotide AS1411 as a novel treatment for cancer. Exp Mol Pathol. 2009; 86: 151-64.

49. Ireson CR, Kelland LR. Discovery and development of anticancer aptamers. Mol Cancer Ther. 2006; 5: 2957-62.

50. Siu LL, Aung KL. Genomically personalized therapy in head and neck cancer. Cancers of the Head \& Neck. 2016; 1.

51. Chithrani DB, Jelveh S, Jalali F, van Prooijen M, Allen C, Bristow RG, et al. Gold nanoparticles as radiation sensitizers in cancer therapy. Radiat Res. 2010; 173: 719-28.

52. Haume K, Rosa S, Grellet S, Smialek MA, Butterworth KT, Solov'yov AV, et al. Gold nanoparticles for cancer radiotherapy: a review. Cancer Nanotechnol. 2016; 7: 8.

53. McQuade C, Al Zaki A, Desai Y, Vido M, Sakhuja T, Cheng Z, et al. A multifunctional nanoplatform for imaging, radiotherapy, and the prediction of therapeutic response. Small. 2015; 11: 834-43.

54. Dam DH, Lee JH, Sisco PN, Co DT, Zhang M, Wasielewski MR, et al. Direct observation of nanoparticle-cancer cell nucleus interactions. ACS Nano. 2012; 6: 3318-26.

55. Adachi M, Cui C, Dodge CT, Bhayani MK, Lai SY. Targeting STAT3 inhibits growth and enhances radiosensitivity in head and neck squamous cell carcinoma. Oral Oncol. 2012; 48: 1220-6.

56. Bonner JA, Trummell HQ, Willey CD, Plants BA, Raisch KP. Inhibition of STAT-3 results in radiosensitization of human squamous cell carcinoma. Radiother Oncol. 2009; 92: 339-44. 